\title{
Calibration of Beta-Particle Ophthalmic Applicators at the National Bureau of Standards
}

\section{J. S. Pruitt}

National Bureau of Standards Gaithersburg, MD 20899

\author{
The method used at the National Bu- \\ reau of Standards for the calibration of \\ strontium-90+yttrium-90 beta-particle \\ ophthalmic applicators in terms of ab- \\ sorbed dose to water, is described. The \\ method involves measurement of ioniza- \\ tion density at the applicator surface \\ with an extrapolation chamber, correc- \\ tion for the difference in backscatter be- \\ tween the collection electrode and \\ water, and application of the Bragg.
}

Gray equation. The calibration obtained is an average over the active surface of the applicator. The overall uncertainty of the surface calibration is about \pm 15 percent.

Key words: absorbed dose; calibration service; electron dosimetry; ophthalmic applicator; surface dose.

Accepted: August 27, 1986

\section{Introduction}

This paper describes the calibration of strontium$90+$ yttrium-90 beta-particle ophthalmic applicators in terms of absorbed-dose rate to water at the surface of the applicator. The calibration is made with an extrapolation ionization chamber, an instrument invented by Failla [1] ${ }^{1}$ which has since been shown by several investigators $[2,3]$ to be particularly well suited to the determination of absorbed-dose rate for beta-particle sources. The techniques of measurement have been sufficiently refined to permit the successful international comparison of several sources at distances of 20 and 30 $\mathrm{cm}$, to within one percent [4].

For ophthalmic applicators, the quantity of interest is absorbed-dose rate at the applicator surface.

About the Author: J. S. Pruitt is with NBS' Ionizing Radiation Division, Center for Radiation Research.

\footnotetext{
${ }^{\prime}$ Figures in brackets indicate literature references.
}

The problems introduced by reducing the source distance from $20 \mathrm{~cm}$ to zero make calibration uncertainties of about 15 percent realistic. Within this limit, it has been found that a reliable surface calibration of an ophthalmic applicator can be obtained with an extrapolation chamber. The extrapolation chamber technique has been used at the National Bureau of Standards (NBS) since 1977 to calibrate about 10 ophthalmic applicators per year. Alternative methods, using thermoluminescent dosimeters (TLD) have also been described [5]. The use of TLD's offers a simpler and less elaborate approach, but it requires calibration of the TLD material in another radiation source, and was not considered an appropriate method for use at NBS.

The general plan of the calibration is to measure ionization current per unit mass of air with the applicator at several distances from the center of the extrapolation chamber air gap, and to extrapolate 
to zero distance to obtain a value at the surface of the applicator. The following three sections are concerned with this determination of extrapolated current per unit mass of air. This is followed by a section describing the calculation of the absorbed. dose rate, and a final section about the errors inherent in this calibration method.

\section{The Extrapolation Chamber}

The extrapolation chamber is essentially the same as that described by Loevinger and Trott [6], with several collecting electrodes which can be interchanged and a thin aluminized-plastic-film highvoltage electrode. The electrode spacing can be changed by rotating the high-voltage-electrode support, at the rate of $0.635 \mathrm{~mm}$ per revolution. The relative position of the electrodes and an ophthalmic applicator under study are shown in figure 1. The particular collecting electrode shown has a diameter of $28 \mathrm{~mm}$ and is surrounded by a guard electrode with an outside diameter of $50 \mathrm{~mm}$. Both guard and collecting electrodes are made of $8 \mathrm{~mm}$ thick A-150 conducting plastic, electrically isolated with $30-\mu \mathrm{m}$ plastic film held in place with epoxy cement. The high-voltage electrode is aluminized plastic with a diameter of $80 \mathrm{~mm}$ and a thickness of $0.7 \mathrm{mg} / \mathrm{cm}^{2}$.

The collecting electrode shown in figure 1 has a much larger area than the active area of all ordinary ophthalmic applicators. This type of collect-

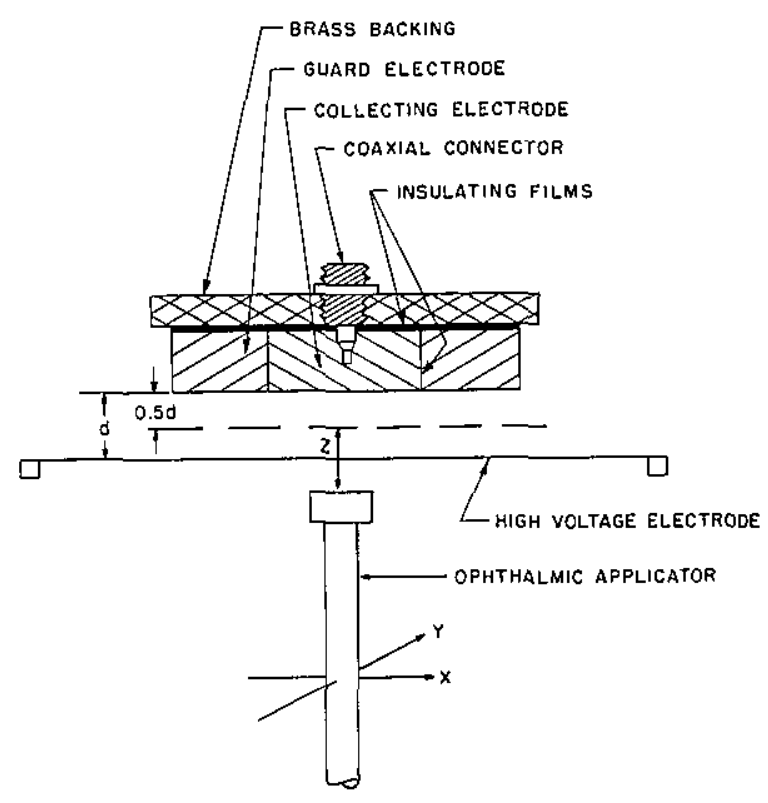

Figure 1-Schematic diagram of ophthalmic applicator and cross section of extrapolation chamber electrodes. ing electrode is used to determine the ratio $(\mathrm{I} / \mathrm{d})_{0}$, where $I$ is the ionization current generated in the air volume defined by the collecting area, $\mathrm{A}$, and the electrode separation, $d$. The ratio $I / d$ is a function of both $d$ and the applicator distance, $z$, and the zero subscript refers to the limiting case where both $d$ and $z$ are zero. This limiting value of $I / d$ is a characteristic of the source and of the electrode material and is independent of $A$, if $A$ is sufficiently large.

A probe electrode of smaller area is used to investigate the variations in dose rate across the surface of applicators. This probe is similar to the unit shown in figure 1 , but the diameter of the collecting electrode is only $0.6 \mathrm{~mm}$, and both collector and guard are made of graphite. A $0.4 \mathrm{~mm}$ thick ring of epoxy between collector and guard results in an effective probe diameter of about $1 \mathrm{~mm}$. With this probe, both the local activity variation and the effective source area are investigated by changing the $x$ and $y$ coordinates of the applicator (fig. 1), for small but fixed values of $z$ and $d$. The applicator stand rests on a microscope stage to permit accurate variation of both $\mathrm{x}$ and $\mathrm{y}$. Accurate adjustment of $\mathrm{z}$ is made with a barrel micrometer.

Extrapolation chamber currents include contributions from (1) ions generated in the collecting volume of the chamber, (2) electrons coming to rest in the collecting electrode, and (3) background. The first of these is the desired quantity. It is separated from (2) and (3) by measuring both $\mathrm{I}^{+}$ with positive high voltage (a positive number) and I- $^{-}$with negative high voltage (a negative number). Since only (1) changes sign with the high voltage, the ion current is $\left(\mathrm{I}^{+}-\mathrm{I}^{-}\right) / 2$, a quantity which is proportional to the electrode separation, to a first approximation. The quantity $\left(\mathrm{I}^{+}+\mathrm{I}^{-}\right) / 2$, called the "parasitic" current by Hillion et al., [7] includes contributions (2) and (3) and is almost independent of electrode separation for a given applicator distance.

Figure 2 shows the variation of both the ion and parasitic currents across the face of the most asymmetric applicator that has been studied in this laboratory. The measurements were made with the probe electrode, using an electrode spacing of 0.05 $\mathrm{mm}$ and an applicator distance of $0.4 \mathrm{~mm} .{ }^{2}$ The asymmetry of the ion current is presumably caused by an uneven distribution of activity across the applicator face. The tails of the ion current distribution are caused by beta rays that leave the applicator with large velocity components perpendicular to the beam direction. The tails rapidly be-

\footnotetext{
${ }^{2}$ In measurements made with the $28 \mathrm{~mm}$ collecting electrode, the parasitic current is only $5-10$ percent of the ion current.
} 


\section{Journal of Research of the National Bureau of Standards}

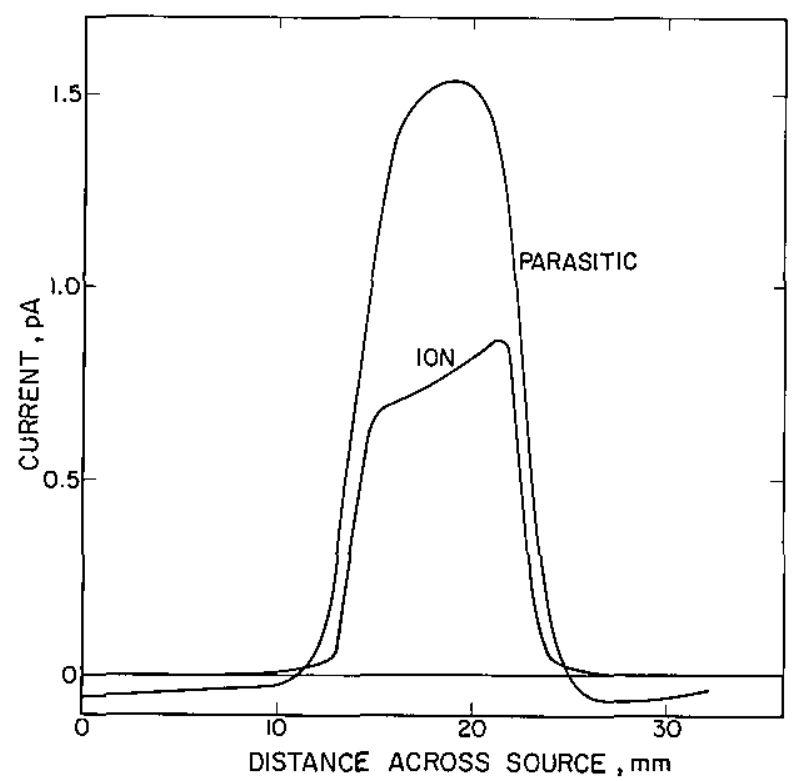

Figure 2-Variation of ion current and parasitic current across applicator SN 0136.

come more prominent as the applicator distance $\mathrm{z}$ increases.

The graph of parasitic current in figure 2 shows evidence both of electrons coming to rest in the collecting electrode (the central peak of the distribution), and background (the negative tails). The central peak of the parasitic current is more symmetrical than the ion current peak, probably because of multiple scattering of the primary beam in the collecting and guard electrodes, which tends to round off the edges of the distribution. The background is a leakage current of about $10-50 \mathrm{fA}$. It is unaffected by high-voltage polarity changes, as stated earlier. The parasitic currents are generally ignored.

\section{Determination of Source Area}

Initial inspection of an applicator is made with the probe electrode, using as small an electrode separation and applicator distance as can be tolerated. The applicator face is mapped at points separated by $2 \mathrm{~mm}$, over a grid of sufficient extent for the ion current to drop below half of its maximum value along all tracks. This crude map permits calculation of the source area and provides a qualitative picture of the source symmetry and activity distribution. On occasion, when more detail is required or when the source diameter is found to be only a few millimeters, a $1 \mathrm{~mm}$ or even a $0.5 \mathrm{~mm}$ grid spacing is used.
The source area is taken to be the area within the locus of half-maximum-current points on such a map. It is determined from a plot of the measured current as a function of radius from the center of the distribution, as shown in figure 3 for a particular applicator. For most applicators, the current decreases monotonically with increasing radius. ${ }^{3}$ A 50 percent radius, $\overline{\mathrm{R}}$, can be read directly from the graph, and the area calculated from this as $\pi \overline{\mathbf{R}}^{2}$. This is a close approximation to the real area, $\pi \mathrm{R}^{2}$, if the chamber spacing, $d$, and source distance, $z$, are both sufficiently small. A rough estimate of uncertainty for a given $\mathrm{d}$ and $\mathrm{z}$ can be obtained from the following equation describing the ionization current per unit volume as a function of position:

$$
\frac{\mathrm{dI}}{\mathrm{dv}}=\mathrm{K} \cdot \log _{\mathrm{e}}
$$

${ }^{3}$ For some applicators, the probe electrode map shows regions of high activity near the edge of the distribution. These hot spots make the determination of area very uncertain and at the same time bring the medical use into question. We decline to calibrate such sources and recommend against their use as ophthalmic applicators.

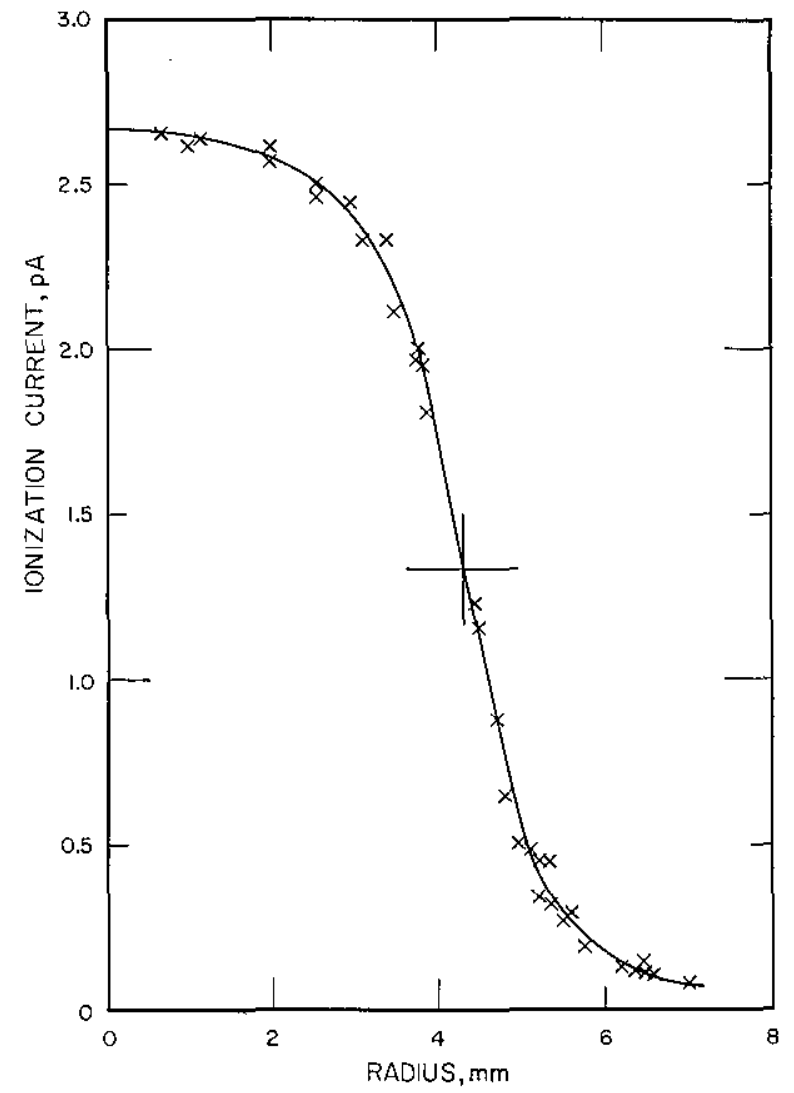

Figure 3-Currents measured with the probe electrode across the face of SN D-402, plotted as a function of radius, measured from the center of the current distribution. The curve was drawn freehand. 


\section{Journal of Research of the National Bureau of Standards}

$$
\left[\frac{1+\eta^{2}-\mu^{2}+\sqrt{1+2\left(\eta^{2}-\mu^{2}\right)+\left(\eta^{2}+\mu^{2}\right)^{2}}}{2 \eta^{2}}\right]
$$

where in terms of the coordinates of figure 1 , $\mu=r / R, \eta=z / R, r=\sqrt{x^{2}+y^{2}}$, and $K$ is a constant.

This equation was derived assuming that the activity is uniformly spread over a circle of radius $R$ on the source surface, that the beta-particles are emitted isotropically from the source surface, that their paths are straight, and that energy deposition is uniform along these paths. The derivation is based on a $1 / \mathrm{r}^{2}$ distance variation and consequently current per unit volume becomes infinite as the distance approaches zero. If the current/volume is normalized to its value on the central axis of the source $(\mu=0)$, the normalized current is well behaved for all distances (that is, for all values of $\eta$ ).

Figure 4 is a plot of the normalized currents for several values of $\eta$. For each curve, the apparent radius, $\vec{R}$, is the product of the true radius, $R$, and the value of $\mu$ where the curve crosses the halfvalue line. For $\eta=1, z=\mathrm{R}$, and $\pi \overline{\mathrm{R}}^{2}$ exceeds $\pi \mathrm{R}^{2}$ by a factor of about 2. For $\eta=0.2$. (not shown) $\pi \overline{\mathrm{R}}^{2}$ exceeds $\pi \mathrm{R}^{2}$ by about 4 percent, and for $\eta=0.1$, the discrepancy is less than 1 percent. In a typical case, $\mathrm{d}=0.2 \mathrm{~mm}, \mathrm{z}=0.12 \mathrm{~mm}, \mathrm{R}=4 \mathrm{~mm}$, and $\eta=0.03$, for which $\pi \overline{\mathrm{R}}^{2} / \pi \mathrm{R}^{2}=1.001$.

Figure 5 is a plot of probe ion current $v$ s. radius for two applicators and matching source profiles calculated from eq (1). The parameter $\mathrm{z}$ was assigned the approximate value $0.1 \mathrm{~mm}$ in the calculation, and values of $\mathrm{R}=3.18$ and $4.35 \mathrm{~mm}$ were read from the graph. All four curves have been

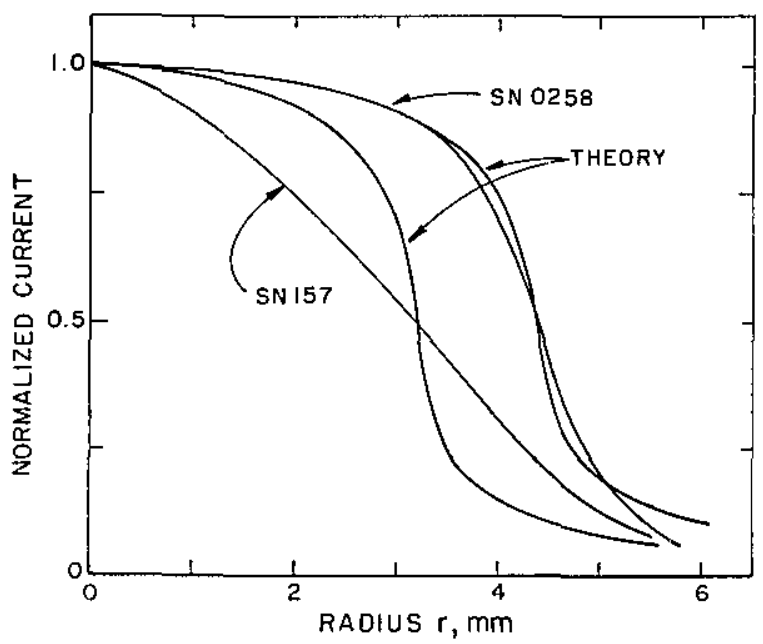

Figure 5-Measured probe profiles of applicators SN 0258 and SN 157, in comparison with theoretical predictions for uniformly distributed sources.

normalized at the origin. Equation (1) is not an accurate description of either source profile, but the comparison with SN 0258 is consistent with the assumption that the activity in this source is spread uniformly over an area of $4.35-\mathrm{mm}$ radius. The small differences between theory and measurement appear to be easily explained. The differences near $r=R$ can be attributed to the finite size of the probe electrode. The differences at larger radii probably arise from the fact that the activity in SN 0258 lies below the applicator face, so that the elemental sources cannot be treated as isotropic at large angles. This will cause the real distribution to fall off more rapidly at large radii than the theoretical dis.

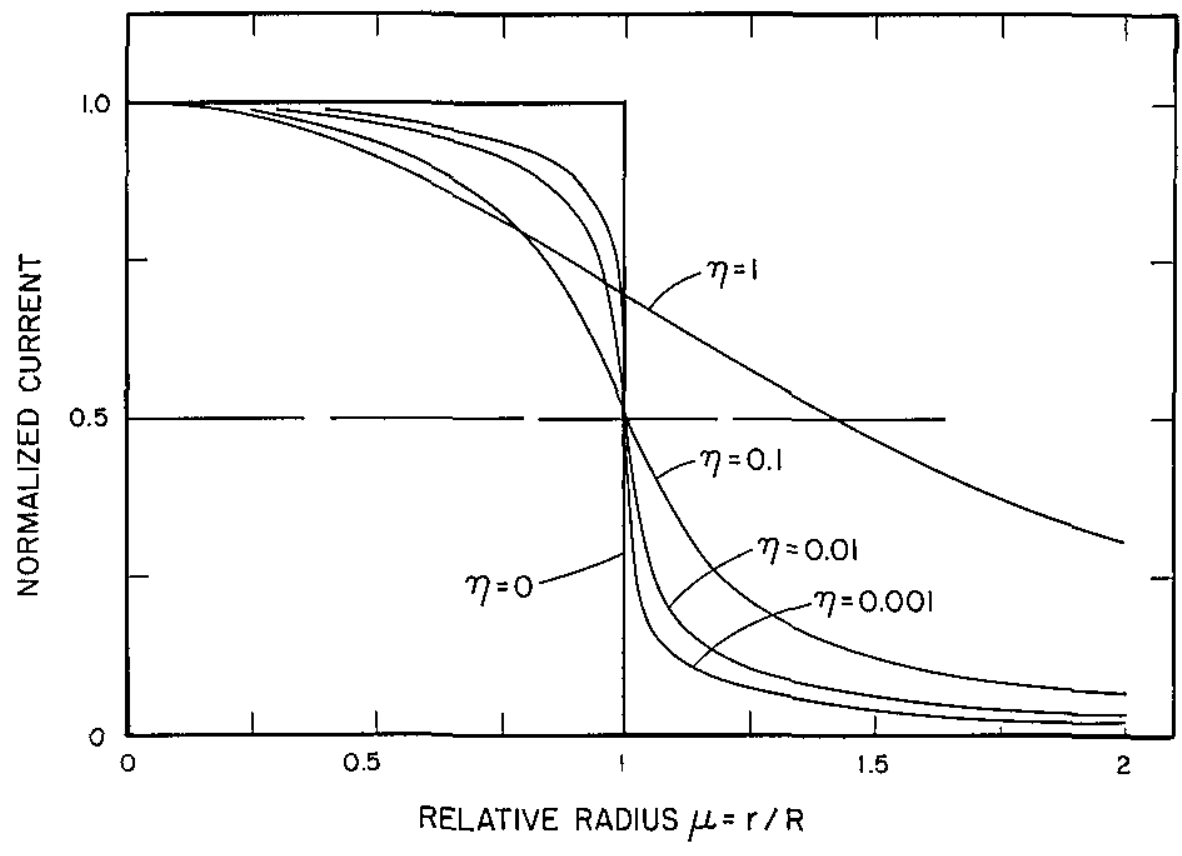

Figure 4-Theoretical probe profiles for five different source distances, $\eta=\mathrm{z} / \mathrm{R}$. 
tribution. The activity in SN 157, in contrast, appears to be highly peaked at the center. ${ }^{4}$

\section{Determination of Average Current Per Unit Mass of Air}

After mapping the source and measuring its area, the probe electrode must be replaced by the larger collecting electrode to determine the current per unit electrode spacing $(\mathrm{I} / \mathrm{d})_{0}$. This is determined by measuring current, I, for five different air-gaps, $\mathrm{d}=0.5,1,1.5,2,2.5 \mathrm{~mm}$, with the source distance $\mathrm{z}=\mathrm{d} / 2$ in each case. These measured currents are corrected (1) to a reference air temperature and pressure, $22{ }^{\circ} \mathrm{C}$ and $101.3 \mathrm{kPa}$; (2) for ion recombination and diffusion, using the formulas and constants summarized by Böhm [8]; and (3) errors in the extrapolation technique, discussed below. The corrected current is a non-linear function of $\mathrm{d}$, because of poor geometry, and extrapolation to zero $\mathrm{d}$ is imprecise. The corrected current is fitted to a cubic equation in d constrained to pass through the origin. The ratio $1 / d$ is then a quadratic function that can be written:

$$
\frac{\mathrm{I}}{\mathrm{d}}=\left(\frac{\mathrm{I}}{\mathrm{d}}\right) \cdot\left(\mathrm{l}+\alpha \mathrm{d}+\beta \mathrm{d}^{2}\right)
$$

In a typical case, $\alpha \approx-0.1 \mathrm{~mm}^{-1}$ and $\beta \approx 0.01$ $\mathrm{mm}^{-2}$.

The ( $/ / d)_{3}$ ratio from eq (2) depends on the extrapolation path to a minor extent. For example, if the ratio $\mathrm{z} / \mathrm{d}$ had been maintained at 1 rather than at $1 / 2$, the measured $(\mathrm{l} / \mathrm{d})_{0}$ ratio would have been slightly smaller. Ideally, the ratio should be measured varying only $d$, with $\mathrm{z}=0$ throughout. The closest one can come to this ideal situation is to extrapolate $I / d$ to zero d for several fixed values of $\mathrm{z}$ and then extrapolate the zero-d limits to zero $\mathrm{z}$ independently, a procedure which increases the calibration time by a large factor. For applicator SN 0258 , it was found that the ideal $(I / d)_{0}$ is 2 percent smaller than the value obtained with $\mathrm{z} / \mathrm{d}=1 /$ 2. This is the origin of the correction factor (3) above, which is set equal to 0.98 .

For a given source, the average current per unit mass of air is the ratio of $(I / d)_{0}$ to source area, $A_{s}$, divided by the air density, $\rho$, at the time of current

${ }^{4} \mathrm{~A}$ closer theoretical fit may be obtained by using $\eta \approx 0.5$ for SN 157 , but this requires the assumption of an unrealistically large $z$.

${ }^{5}$ No corrections were made for the foil deformation effects discussed by Loevinger. These effects are too small to be detected with the Mylar foils used. measurement. Since the currents have been corrected to the reference temperature and pressure, the relevant density is $1.197 \mathrm{~kg} / \mathrm{m}^{3}$.

\section{Calculation of Absorbed Dose}

The average surface absorbed-dose rate in water is given by a variant of the Bragg-Gray equation:

$$
\mathbf{D}_{\text {water }}=\frac{(\mathrm{L} / \mathrm{d})_{0}}{\rho \mathrm{A}_{\mathrm{s}}}(\mathrm{W} / \mathrm{e})(\overline{\mathrm{S}} / \rho)_{\mathrm{air}}^{\mathrm{waber}} \mathbf{k}_{\mathrm{tack}} \mathrm{k}_{\mathrm{fcil}}
$$

where $\mathrm{W} / \mathrm{e}$ is the average energy required to produce unit charge in ambient air, $(\overline{\mathrm{S}} / p)_{\text {air }}^{\text {water }}$ is the ratio of the average collision mass stopping power of water to that of air, $\mathrm{k}_{\text {tack }}$ corrects for a difference in backscatter between the collecting electrode and water, and $\mathrm{k}_{\text {foil }}$ corrects for attenuation or buildup in the high-voltage electrode.

The value, $w / e=33.7 \mathrm{~J} / \mathrm{C}$, is used in eq (3) [9]. The stopping power ratio was calculated with unrestricted stopping powers [10], using two different source spectra. The first spectrum was based on unfiltered theoretical spectra for strontium 90 and yttrium 90 [11], modified by the inherent filtration in a particular source, SN 0258, and yielded the value $(\overline{\mathrm{S}} / \rho)_{\mathrm{arr}}^{\mathrm{water}}=1.123$. The second spectrum was measured with a lithium-drifted germanium detector for a strontium-yttrium source filtered by 50 $\mathrm{mg} / \mathrm{cm}^{2}$ of silver and $0.1 \mathrm{~mm}$ of steel and yielded the value $(\overline{\mathrm{S}} / \rho)_{\mathrm{air}}^{\text {water }}=1.124$, the value used in our calibrations. ${ }^{\circ}$

The backscatter factor was estimated from published formulas for backscatter probability as a function of atomic number and beta-particle energy [12]. Using the chemical formulation for the collecting electrode material, and the second source spectrum described above, the value $k_{\text {back }}=1.010$ was obtained.

The foil attenuation correction factor, $\mathrm{k}_{\text {foil }}=1.003$, was determined from measurements with extra thicknesses of foil.

In summary, the four right-hand factors in eq (3) may be combined to form a single factor, 38.4 Gy.kg/C. Multiplication of this factor by the ratio of current per unit mass determined with the techniques of the previous section gives average absorbed-dose rate to water over the active surface of the applicator.

\footnotetext{
${ }^{6}$ The value $\left.(\overline{\mathrm{S}} / \rho)\right)_{\text {air }}^{\text {wacr }}=1.15$, based on earlier stopping-power tabulations, had been used until recently.
} 


\section{Calibration Uncertainty}

There is some uncertainty associated with each term of eq (3). Those determined by extrapolationchamber measurements with the $28 \mathrm{~mm}$ diameter collecting electrode, such as $(\mathrm{I} / \mathrm{d})_{0}$ and $\mathrm{k}_{\text {foil, }}$ have statistical uncertainties of the order of 0.1 percent. These are much smaller than the estimated uncertainties and are usually ignored. The estimated uncertainties are listed in table 1. Each is intended to be comparable to a standard deviation, in that the chance that the true value of the quantity lies within plus or minus the stated uncertainty is intended to be about 2 out of 3 .

The instrumental uncertainties include voltmeter readings, capacitance measurements, and pressure and temperature determination. The uncertainty in W/e comes directly from reference 9. The stopping-power-ratio uncertainty includes uncertainties in the stopping-powers themselves plus uncertainties in the beta-particle spectra. The uncertainty in $(\mathrm{I} / \mathrm{d})_{0}$ is an estimate of the reliability of a cubic fit to an extrapolation curve with $z=d / 2$, and the corrections (1), (2), and (3) in the text. The uncertainty in surface area is a standard deviation, based on repeat measurements for several sources, using the normal 2-mm grid.

When the uncertainties are combined in quadrature to obtain a combined uncertainty, the result is 7 percent. This number is intended to have the ap-

Table 1. Estimated uncertainties.

\begin{tabular}{lc}
\hline Absorbed-dose-rate uncertainties & $(\%)$ \\
\hline Instrumental & 0.3 \\
Average energy per ion pair, w/e & 0.4 \\
Stopping-power ratio, (S $/ \rho)_{\text {air }}^{\text {ater }}$ & 3 \\
Rate of change of current, (I/d) & 3 \\
Backscatter correction, k kack & 1 \\
Attenuation correction, $\mathrm{k}_{\text {foil }}$ & $<0.1$ \\
Source surface area, As & 6 \\
Combined uncertainty (quadratic sum)
\end{tabular}

proximate character of a standard deviation. Doubling it gives an overall uncertainty of 14 percent which can be interpreted as having the approximate character of a 95 percent confidence limit. For convenience, this uncertainty is rounded off to 15 percent.

The surface area uncertainty is dominant in table 1 and is the only one which can be appreciably reduced at present. It can be reduced to about I percent by mapping a normal source with a $1-\mathrm{mm}$ grid, but this step is so much more time-consuming that it is not offered as an NBS calibration service. In this case, however, the combined overall uncertainty would be about 4 percent. This would lead to a stated uncertainty of 8 percent with the approximate character of a 95 percent confidence limit.

As a more practical alternative, it has been suggested that the source surface area could be determined with greater accuracy with radiochromic dye films.

\section{References}

[1] Failla, G., Radiology 29, 202 (1937).

[2] Wahlberg, T., Acta Radiol. 30, 201 (1948).

[3] Loevinger, R., Rev. Sci. Inst. 24, 907 (1953).

[4] Böhm, J.; P. Hillion and J. P. Simoen, VIII ${ }^{e}$ Congres International de la Societé Francaise de Radioprotection (1976).

[5] Hendee, W. R., Measurement and correction of nonuniform surface dose rates from beta eye applicators, Am. J. Roent. 103, 734 (1968).

[6] Loevinger, R., and N. G. Trott, Int. J. Appl. Rad. Isotopes 17, 103 (1966).

[7] Hillion, P.; J. P. Simoen and J. P. Guiho, VIII ${ }^{e}$ Congres International de la Societé Francaise de Radioprotection (1976).

[8] Böhm, J., Phys. Med. Biol. 21, 754 (1976).

[9] ICRU Report 31, Average Energy Required to Produce an Ion Pair, International Commission on Radiation Units and Measurements (Washington, DC, 1979). The value of $\mathrm{W} / \mathrm{e}$ cited comes from the value for dry air $(33.85 \mathrm{~J} / \mathrm{C})$, modified by a humidity factor for ambient air taken from figure 5.14 .

[10] Berger, M., and S. Seltzer, Stopping Powers and Ranges of Electrons and Positrons, National Bureau of Standards Internal Report, NBSIR 82-2550A (1983); also as ICRU Report 37 (1984).

[11] Mantel, J., Int. J. A.ppl. Rad. Isotopes 23, 407 (1972).

[12] Tabata, $T$; R. lto and $S$. Okabe, An empirical equation for the backscattering coefficient of electrons, Nucl. Inst. Methods 94, 509 (1971). 